Cinémas

Revue d'études cinématographiques

Journal of Film Studies

\title{
Élise Domenach, Stanley Cavell, le cinéma et le scepticisme, Paris, Presses universitaires de France, 2011, 176 p.
}

\section{Pierre-Alexandre Fradet}

Volume 25, numéro 1, automne 2014

URI : https://id.erudit.org/iderudit/1030235ar

DOI : https://doi.org/10.7202/1030235ar

Aller au sommaire du numéro

Éditeur(s)

Cinémas

ISSN

1181-6945 (imprimé)

1705-6500 (numérique)

Découvrir la revue

Citer ce compte rendu

Fradet, P.-A. (2014). Compte rendu de [Élise Domenach, Stanley Cavell, le cinéma et le scepticisme, Paris, Presses universitaires de France, 2011, 176 p.] Cinémas, 25(1), 179-188. https://doi.org/10.7202/1030235ar d'utilisation que vous pouvez consulter en ligne.

https://apropos.erudit.org/fr/usagers/politique-dutilisation/ 


\section{Élise Domenach, Stanley Cavell, le cinéma et le scepticisme, Paris, Presses universitaires de France, 2011, 176 p.}

Élise Domenach est maître de conférences en études cinématographiques à l'École normale supérieure de Lyon et membre du comité de rédaction de deux revues d'importance dans le paysage intellectuel, Positif et Esprit. Nous attarder ici à sa première monographie, qui a déjà suscité en France un certain nombre de recensions, ne permettra pas que de redire en quoi son ouvrage enrichit de façon notable les études cavelliennes; cela contribuera aussi - tel est du moins notre dessein — à diffuser en sol québécois la pensée d'un auteur américain qui, ironiquement, est plus connu en France que dans l'Amérique française, et à dégager quelques pistes de réflexion sur les croisements possibles, mais insoupçonnés, entre l'œuvre cavellienne et le cinéma québécois.

Le premier des nombreux mérites du livre de Domenach tient à son objectif central, formulé dès l'incipit: interroger «les premières années de [1]a production philosophique [de Cavell], le premier temps de sa réinterprétation du scepticisme fixé dans un "trio" de livres: Dire et vouloir dire (1969), La projection du monde (1971) et Sens de Walden (1972)». On a souvent mentionné, et Stanley Cavell (2001, p. 39) l'a luimême confirmé, que les thèmes de prédilection du philosophe se trouvent préfigurés dès le début de sa réflexion. Ainsi Sandra Laugier (2009, p. 7-8) fait-elle observer qu' " on peut tirer rétrospectivement toute son ouvre [...] de [s]es premiers écrits, qui ont posé sa voix comme singulière et inimitable». Aussi connue que soit cette préfiguration, c'est à Domenach, précisément, qu'il revient d'avoir expliqué en détail comment la plupart des préoccupations de Cavell hantent déjà ses premières œuvres. 
Lauteure rappelle tout d'abord que la visée du philosophe est moins de se pencher sur l'essence du cinéma et de proposer une philosophie du cinéma que de voir de quoi est capable philosophiquement le septième art pour nous, dans nos vies, en tant qu' «éducateur des adultes». Non pas que Cavell renonce à forger une ontologie du cinéma (expression qu'il emploie volontiers lui-même lorsqu'il définit les spécificités du médium); mais il examine ce qui fait du cinéma un art important à nos yeux et pertinent aux fins de notre perfectionnement moral. En cela, le professeur de Harvard nous invite à nous détourner de la question de l'auteur et du mode de production technique, afin de repartir de la position du spectateur. Quelle importance le septième art peut-il avoir pour nous? Comment nous amène-t-il à transformer nos vies ${ }^{1}$ ?

Lun des nerfs moteurs de la réflexion cavellienne est le problème, majeur entre tous, du scepticisme. Avant d'aborder cette question de front, Domenach se penche sur la catégorie critique de médium et contredit l'interprétation de Noël Carroll selon laquelle Cavell, à l'instar de Bazin, estime que le film reproduit automatiquement le passé. Non, Cavell ne se borne pas à défendre une ontologie réaliste du médium cinématographique: il complique ce champ d'interrogation au moyen de catégories nouvelles. Alliant une réflexion sur l'ontologie à "une enquête sur la manière dont certains films donnent sens et importance (significance) aux possibilités et aux propriétés du médium " (p. 89), Cavell s'interroge à la fois sur le mode de présence des objets au cinéma et sur les possibilités esthétiques du médium telles qu'elles s'expriment dans les ouvres elles-mêmes. Ici, la question n'est plus de savoir si le cinéma représente ou non la réalité, mais plutôt: «qu’arrive-t-il à la réalité quand elle est projetée sur un écran?» (p. 94). Cavell remplace dès lors la question de la représentation par celle de la projection.

Dans un chapitre qui a pour titre «L'expression du scepticisme au cinéma ", Domenach reprend et approfondit cette catégorie de projection en précisant sous quel angle le scepticisme est abordé par Cavell. Là se repère d'ailleurs le second des grands mérites de l'ouvrage: l'auteure y met en lumière, dans une langue épurée et fluide, la distinction entre scepticisme intellectuel et scepticisme 
vécu, et éclaircit du même coup l'un des traits les plus notables de la pensée cavellienne. Le scepticisme intellectuel désigne "toute conception qui tient l'existence du monde pour un problème de connaissance» (Cavell 1979, p. 88). Le scepticisme vécu, quant à lui, renvoie à "l'impossibilité de la conversation, l'impossibilité d'entrer en relation avec autrui dans le langage: impossibilité mise en scène dans la tragédie, mais qui est notre lot quotidien" (Laugier 2001, p. 105). En dégageant et en étudiant méthodiquement ces deux formes de scepticisme, Domenach note un singulier déplacement dans la pensée cavellienne. Chez Cavell, le scepticisme ne désigne pas qu'une mise en doute de la réalité du monde (comme c'est le cas dans la tradition philosophique en général); il se rapporte à tout questionnement sur notre accès au monde et à certains pans de l'expérience ordinaire.

C'est un lieu commun de dire que les philosophes du langage ordinaire résolvent le problème sceptique en refusant de le prendre au sérieux. Tandis que les sceptiques nous somment de produire une preuve de la réalité du monde, Wittgenstein et Strawson, pour ne mentionner qu'eux, discréditent cette exigence même: l'un "ram[ène] les mots de leur usage métaphysique à leur usage ordinaire» (Wittgenstein 1953, \$116) et met en évidence le non-sens qui pointe à l'horizon de la position sceptique; l'autre relève l'incohérence des doutes sceptiques qui, en s'élevant au-dessus du langage ordinaire, "équivalent à un rejet de tout le système conceptuel à l'intérieur duquel seul de tels doutes ont un sens» (Strawson 1959, p. $38^{2}$ ). Or, tout en congédiant le scepticisme intellectuel et en reconnaissant que les tentatives de nier le problème sceptique en constituent des manifestations, Cavell (1989, p. 56) affirme que le septième art a le pouvoir de rejoindre une vaste part de la population pour lui exprimer sa condition sceptique et lui apprendre à la domestiquer.

Alors que «scepticisme et tragédie concluent tous deux que la condition humaine est la séparation» (Cavell 1979, p. 560), les comédies hollywoodiennes du remariage nous invitent à vaincre notre scepticisme en mettant en scène deux amants qui font l'expérience d'un éloignement puis d'un rapprochement ${ }^{3}$. Elles nous demandent de nouer ou de renouer le contact avec nos semblables par la conversation - exigence difficile, dans la 
mesure où elle nous impose de sortir de notre état d'exil et de supporter sans relâche le poids de la signification et de l'expression $^{4}$. Cette sortie de l'isolement est censée permettre à l'individu de rester fidèle à son moi et de s'engager avec autrui dans des exercices de reconnaissance mutuelle. Le médium cinématographique a lui-même quelque chose à dire au sujet du scepticisme. D'un côté, paradoxalement, il nous met en face d'une réalité qui n'est pas là — «la présence des objets à l'écran renvoie à leur absence» (p. 144) —, de sorte que le réel projeté à l'écran semble toujours sur le point de se perdre ou d'être relégué au statut d'illusion. De l'autre, ce médium a le pouvoir "d'animer le monde en nous réduisant à la passivité, à l'impuissance; de nous "absorber" dans le spectacle de l' "être-là" des choses» (p. 157). C'est que l'expérience de projection a ceci de particulier qu'elle nous replace dans un état où nous retrouvons automatiquement le réel en dehors de toute intervention subjective. Plongés dans l'obscurité, immobiles et passifs, nous croyons avoir affaire à «la monstration du monde en soi » (p. 133). Cette passivité nous amène à réinvestir une part de notre expérience vécue, "moments banals où se tisse notre proximité ordinaire avec le monde" (p. 146); elle nous incline à accueillir le réel comme un don sans obligation de preuve.

On comprend dès lors que la question de l'ordinaire traverse plusieurs niveaux chez Cavell, qui fait simultanément référence au langage ordinaire et à deux versants de l'expérience ordinaire: celui où l'on est mis à l'épreuve par le scepticisme vécu et celui où l'on renoue contact avec le monde par la passivité. L'expérience ordinaire ne constitue donc pas pour lui un espace homogène: elle appelle une attention critique et comporte au minimum deux pans distincts. Expression à multiples sens chez Cavell, l'ordinaire s'intrique à la fois aux notions de quotidien (everyday) et de bas (low) sans pourtant se confondre avec elles. Il est souvent rattaché à une posture, à une condition ou à un certain usage dont témoigne l'expérience vécue en communauté - ce qui permet selon nous de rapprocher l'ordinaire cavellien de l'acception pratique et normative du sens commun, bien que le philosophe prenne ses distances d'avec le concept de sens commun entendu comme croyance naïve. 
Le troisième des grands mérites de l'ouvrage de Domenach découle des liens que tisse l'auteure entre la pensée de Cavell et un corpus théorique et filmique. Dans des passages denses mais lumineux, elle traite du rapport qu'entretient Cavell avec John Austin, Michael Fried, Clement Greenberg (autour de la question du modernisme) et fait référence à des cinéastes comme Frank Capra, Terrence Malick, Woody Allen, Buster Keaton, Éric Rohmer, Robert Gardner, George Cukor, Stan Brakhage, Jonas Mekas... Il serait impossible de rendre ici justice à la totalité de ses analyses. Ce qu'il est loisible de faire, en revanche, c'est d'évoquer de façon programmatique un ensemble d'objets et de films à mettre éventuellement en rapport avec la pensée de Cavell. Non pas qu'on veuille cibler ici une carence dans l'ouvrage de Domenach. Mais il pourrait être bon, en complément d'analyse, d'établir des liens entre la pensée de Cavell et des champs avec lesquels on la fait moins souvent dialoguer: la télévision, la philosophie continentale ${ }^{5}$ et "post-continentale" (incluant les représentants du tournant spéculatif intéressés par la chose en soi), de même que les films qui au premier abord ne semblent pas directement rejoindre les préoccupations cavelliennes. Ce travail d'élargissement comparatif semble d'autant plus souhaitable que la pensée de Cavell est souvent abordée à travers le prisme analytique, tandis qu'elle se réclame de Wittgenstein et du transcendantalisme d'Emerson et de Thoreau, s'inspire de Shakespeare et ne peut être réduite ni au versant analytique ni au versant continental de la philosophie.

Trop souvent tenus à l'écart de la pensée en général et de la philosophie en particulier ${ }^{6}$, les films québécois issus du cinéma direct, du cinéma autochtone et de ce qu'on a appelé le «renouveau du cinéma québécois» - ainsi que d'autres ouvres du corpus largement placé sous le signe de la question de l'ordinaire $^{7}$ - pourraient alimenter des discussions sur ce thème. Il est bien connu que les cinéastes du direct ont privilégié les tournages légers et tendu la perche au commun des mortels. Ils ont ainsi non seulement révolutionné la technique cinématographique, mais engagé une réflexion pénétrante sur l'accès au réel et le langage ordinaire. Parmi une constellation d'interrogations possibles, se pose, entre autres, la question de savoir si le cinéma 
direct n'a pas pour conséquence d'exprimer le scepticisme vécu, voire de le conjurer. En permettant à tous de prendre la parole dans le cadre de leurs activités quotidiennes, ce cinéma semble en effet susceptible de dévoiler des particularités de l'expérience ordinaire et de favoriser l'activité d'expression dans des exercices de reconnaissance mutuelle.

Quant aux autochtones, ils sont de plus en plus nombreux à passer derrière la caméra depuis quelques décennies. Il suffit de mentionner Zacharias Kunuk, Alanis Obomsawin, Diane Obomsawin, Yves Sioui Durand, Bobby Kenuajuak, Jobie Weetaluktuk, Natar Ungalaaq, Mike Jaypoody, Kevin Papatie, Alethea Arnaquq-Baril ${ }^{8}$... Plusieurs d'entre eux mettent en images leur propre vécu, tantôt pour dénoncer les conditions de vie dans lesquelles ils évoluent, tantôt pour proposer de leurs communautés une vision autre que le traitement misérabiliste qui leur est le plus souvent réservé. À des fins de dénomination, on pourrait qualifier ce corpus filmique de cinéma "autoamérindien" — ou "auto-autochtone», l'intention étant d'inclure également les œuvres créées par les Métis et les Inuits. Par ces néologismes qui font écho à un ouvrage de Georges Sioui (1989), professeur à l'Université d'Ottawa, il faut entendre les films réalisés en Amérique du Nord par des autochtones et dont l'un des sujets cardinaux est la vie autochtone elle-même. Mettre en parallèle les œuvres auto-autochtones et la pensée cavellienne impliquerait bien entendu d'être attentif à des conceptions du sens commun irréductibles à celles dont est dépositaire la tradition occidentale.

Un autre corpus cinématographique pourrait éveiller des réflexions fertiles au sujet de l'expérience ordinaire: le renouveau du cinéma québécois, aussi appelé "nouvelle vague» ou «mouvée» (Sirois-Trahan 2010). Mis à part Rafaël Ouellet (voir Fradet 2014a), aucun cinéaste ou presque ne se réclame sans détour du renouveau du cinéma québécois, ce qui s'explique en partie par le fait que la plupart des réalisateurs veulent éviter de se sentir réduits à un courant assignable. Il n'en demeure pas moins que certains «airs de famille» entre films permettent de repérer plus que le semblant d'un renouveau dans la production actuelle: un tournage parfois léger, une diminution du nombre 
de plans, un souci d'aborder les questions politiques de biais plutôt que de front, un désir de faire voyager ses films à l'étranger, une capacité à créer à partir de budgets limités, un intérêt marqué pour les milieux ruraux et les lieux désolés, une fascination pour l'ordinaire et une exaltation de la lenteur - autant de traits qui n'impliquent pas, loin de là, que les cinéastes du renouveau se contentent d'exprimer de façon convenue la durée bergsonienne ${ }^{9}$.

Relativement au problème de l'ordinaire, plusieurs des œuvres du renouveau approfondissent le thème du scepticisme vécu. C'est le cas par exemple de J'ai tué ma mère (Xavier Dolan, 2009), de Nuages sur la ville (Simon Galiero, 2009) et de Laurentie (Mathieu Denis et Simon Lavoie, 2011). J'ai tué ma mère met en scène un jeune homme et sa mère dont les liens sont rompus, mais qui, au terme d'un cheminement houleux, parviennent à se réconcilier. Nuages sur la ville porte avant tout sur la place qu'occupe le has-been en société, mais met aussi en évidence un phénomène de déconnexion intergénérationnelle (un homme d'âge mûr demandant à son fils, encore jeune mais déjà trop vieux pour s'adonner à ce genre de loisir, s'il veut «jouer au ballon»). Laurentie ancre pour sa part son discours dans le contexte politique québécois. Loin de se contenter d'offrir "un plaidoyer souverainiste» (Gendreau 2012, p. 57) et d'accuser les anglophones d'agir en envahisseurs et les francophones de se complaire dans la passivité, l'impuissance, l'abattement, l'œuvre raconte la vie d'un francophone en proie à une rage croissante du fait de son incapacité à entrer en contact avec ses voisins anglophones, incapacité dont il se rend lui-même (en partie) responsable. Laurentie évoque ainsi une forme singulière de scepticisme, qui fait écho au contexte politique de l'Amérique française.

Autre créateur, autre traitement: le cas de Denis Côté. Au premier abord, tout semble indiquer que ses œuvres n'ont rien à voir avec l'analyse cavellienne. Alors que Cavell suggère que chacun de nous éprouve, à un moment ou à un autre de son existence, un sentiment d'isolement, les films de Denis Côté présentent des individus étouffés par leurs liens sociaux et avides d'éloignement. Ce qui constitue un point de départ chez Cavell, la distance 
entre les individus, paraît devenir une fin à atteindre chez Côté. Le regard que porte Côté sur la société n'implique cependant pas que les individus vivent ou doivent vivre en vase clos, sur un mode insulaire; il suggère que la société suppose déjà une certaine connexion, mais une connexion déficiente, maladive, chancelante, dont les failles commandent l'établissement de nouveaux rapports (Froger 2010). On peut voir là un diagnostic complémentaire de l'analyse cavellienne au sujet du scepticisme vécu, qui donne à penser que la réflexion philosophique québécoise, loin de se manifester uniquement par écrit (Fernand Dumont, Charles Taylor, Jacques Lavigne, Charles De Koninck, Thomas De Koninck, Michel Freitag, Jean Grondin...), s'exprime aussi de façon singulière dans le cinéma lui-même.

De plus en plus nombreux sont ceux qui forment la communauté des lecteurs de Cavell en France. Or, grâce aux stimulants travaux de philosophes, commentateurs et traducteurs comme Élise Domenach, Sandra Laugier, Christian Fournier et Hugo Clémot, il est à espérer que cette communauté aille aussi grandissant dans le reste du monde francophone, tout comme on peut souhaiter qu'un nombre croissant d'études tracent des parallèles inédits entre l'œuvre cavellienne et un ensemble d'œuvres filmiques, dont celles du cinéma québécois. Il faut bien sûr approuver le souci des chercheurs d'aborder la philosophie de Cavell à partir des textes originaux et reconnaître l'excellence des travaux anglais portant sur la philosophie cavellienne (Stephen Mulhall, David Rodowick, Andrew Norris, William Rothman...). Mais il importe de saluer parallèlement l'entreprise de traduction française des textes de Cavell et d'inciter les chercheurs francophones d'Amérique à se pencher avec plus d'insistance sur ce penseur incontournable. Car c'est grâce aux efforts de traduction que la pensée cavellienne peut circuler auprès d'un vaste public et, en partie, grâce aux travaux critiques rédigés en français que la réception de Cavell peut être renouvelée, en sol américain comme ailleurs.

Pierre-Alexandre Fradet

ENS de Lyon / Université Laval 


\section{NOTES}

1. Voir par exemple Cavell 2003.

2. Dans le sillage de Cora Diamond (1991) et à l'encontre des interprétations conventionnalistes de Wittgenstein (Kripke 1982; Seymour 2005), Sandra Laugier (2005, p. 99 et suiv.) a beaucoup insisté sur l'aspect naturel des formes de vie où se construisent les conventions linguistiques afin de faire ressortir le caractère non arbitraire du langage ordinaire.

3. Sur le sujet, voir entre autres Curley 1989.

4. Voir notamment Laugier 1999, p. 146.

5. Voir par exemple Rodowick s. d., Santini 2004 et Gallope 2010. Nous entendons nous-mêmes approfondir ailleurs le programme de recherche ouvert ici.

6. Voir par exemple le considérable ouvrage de De Baecque et Chevallier (2012).

7. Voir Poirier 2004, p. 74.

8. Voir notamment Lewis 2006, Cornellier 2011 et Evans 2008.

9. Sur les différents traits de la durée, voir Fradet 2014 b.

\section{RÉFÉRENCES BIBLIOGRAPHIQUES}

Cavell 1979: Stanley Cavell, Les voix de la raison. Wittgenstein, le scepticisme, la moralité et la tragédie [1979], traduit de l'anglais par Sandra Laugier et Nicole Balso, Paris, Seuil, 1996, 720 p.

Cavell 1989: Stanley Cavell, Une nouvelle Amérique encore inapprochable. De Wittgenstein à Emerson [1989], traduit de l'anglais par Sandra Laugier, Combas, L'Éclat, 1991, repris dans Qu'est-ce que la philosophie américaine?, Paris, Gallimard, 2009, p. 1-151.

Cavell 2001 : Stanley Cavell, «Nouvelle préface de l'auteur à l'édition de 2002 », dans Stanley Cavell, Dire et vouloir dire, traduit de l'anglais par Sandra Laugier et Christian Fournier, Paris, Cerf, 2009, p. 39-55.

Cavell 2003: Stanley Cavell, Le cinéma nous rend-il meilleurs?, textes rassemblés par Élise Domenach et traduits de l'anglais par Christian Fournier et Élise Domenach, Paris, Bayard, 2003, 218 p.

Cornellier 2011 : Bruno Cornellier, "La "chose indienne" : cinéma et politiques de la représentation autochtone dans la colonie de peuplement libérale", Thèse de doctorat, Université Concordia, 2011.

Curley 1989: Edwin Curley, "Cavell and the Comedy of Remarriage», Philosophy Research Archives, vol. 14, 1988-1989, p. 581-603.

De Baecque et Chevallier 2012: Antoine de Baecque et Philippe Chevallier (dir.), Dictionnaire de la pensée du cinéma, Paris, PUF, 2012, 792 p.

Diamond 1991 : Cora Diamond, L'esprit réaliste. Wittgenstein, la philosophie et l'esprit [1991], traduit de l'anglais par Emmanuel Halais et Jean-Yves Mondon, Paris, PUF, 2004, 524 p.

Evans 2008: Michael Robert Evans, Isuma: Inuit Video Art, Montréal/Kingston, McGill-Queen's University Press, 2008, 236 p.

Fradet 2014a : Pierre-Alexandre Fradet, «Entretien avec Rafaël Ouellet: le néoterroir au cinéma", Spirale, n 250 , p. 37-40.

Fradet 2014b : Pierre-Alexandre Fradet, Derrida-Bergson. Sur l'immédiateté, Paris, Hermann, 2014, $234 \mathrm{p}$.

Froger 2010 : Marion Froger, "Réflexivité et socialité dans Les états nordiques», Revue canadienne d'études cinématographiques, vol. 19, n 1, 2010, p. 59-74. 
Gallope 2010 : Michael Gallope, "Cavell and Deleuze», Journal of Music Theory, vol. 54, no 1, 2010, p. 107-120.

Gendreau 2012: Philippe Gendreau, "Maintenir le déséquilibre. Rencontre avec Simon Lavoie et Mathieu Denis ", Liberté, vol. 54, nº 1 (297), 2012, p. 54-58.

Kripke 1982: Saul A. Kripke, Wittgenstein on Rules and Private Language, Cambridge, Harvard University Press, 1982, 150 p.

Laugier 1999: Sandra Laugier, Du réel à l'ordinaire. Quelle philosophie du langage aujourd'hui?, Paris, Vrin, 1999, 173 p.

Laugier 2001 : Sandra Laugier, "La comédie du remariage comme philosophie américaine ", dans Sandra Laugier et Marc Cerisuelo (dir.), Stanley Cavell. Cinéma et philosophie, Paris, Presses de la Sorbonne Nouvelle, 2001, p. 95-111.

Laugier 2005: Sandra Laugier, "Arguments transcendantaux et limites du sens", dans Jocelyn Benoist et Sandra Laugier (dir.), Langage ordinaire et métaphysique. Strawson, Paris, Vrin, 2005, p. 85-116.

Laugier 2009: Sandra Laugier, "Présentation», dans Stanley Cavell, Dire et vouloir dire, traduit de l'anglais par Sandra Laugier et Christian Fournier, Paris, Cerf, 2009, p. 7-37.

Lewis 2006: Randolph Lewis, Alanis Obomsawin: The Vision of a Native Filmmaker, Lincoln, University of Nebraska Press, 2006, 260 p.

Poirier 2004: Christian Poirier, Le cinéma québécois. À la recherche d'une identité?, Tome 1, Limaginaire filmique, Sainte-Foy, Presses de l'Université du Québec, 2004, $314 \mathrm{p}$.

Rodowick s. d. : David Norman Rodowick, "Ethics in Film Philosophy (Cavell, Deleuze, Levinas)», s. d., http://isites.harvard.edu/fs/docs/icb.topic242308.files/ RodowickETHICSweb.pdf.

Santini 2004: Sylvano Santini, "Le pari transcendantal de Cavell dans le contexte américain", Spirale, n 197, 2004, p. 41-42.

Seymour 2005: Michel Seymour, L'institution du langage, Montréal, Presses de l'Université de Montréal, 2005, 446 p.

Sioui 1989: Georges Emery Sioui, Pour une autohistoire amérindienne. Essai sur les fondements d'une morale sociale, préface de Bruce G. Trigger, Québec, Presses de l'Université Laval, 1989, 157 p.

Sirois-Trahan 2010: Jean-Pierre Sirois-Trahan, «La mouvée et son dehors: renouveau du cinéma québécois", Cahiers du cinéma, n 660, 2010, p. 76-78.

Strawson 1959: Peter Frederick Strawson, Les individus. Essai de métaphysique descriptive [1959], traduit de l'anglais par Albert Shalom et Paul Drong, Paris, Seuil, 1973, 287 p.

Wittgenstein 1953: Ludwig Wittgenstein, Recherches philosophiques [1953], traduit de l'allemand par Élisabeth Rigal et al., Paris, Gallimard, 2005, 367 p. 